\title{
Démantèlement des installations nucléaires : une faisabilité technique pas tout à fait acquise
}

Barbara Romagnan, députée (2012-2017)

\section{Le choix de procéder}

au démantèlement

des installations nucléaires

après leur arrêt devrait

permettre de réutiliser

les espaces libérés.

Cependant, la faisabilité

technique des opérations

de déconstruction

et décontamination n'a pas

encore été démontrée sur

la diversité du parc français.

\section{En savoir plus}

- Rapport sur le démantèlement des installations nucléaires de base, Conseil supérieur de la sureté et de l'information nucléaires (CSSIN), 16 mai 2007.

- Faisabilité technique et financière du démantèlement des infrastructures nucléaires, rapport de la Mission d'information parlementaire (M. Julien Aubert, président, Mme Barbara Romagnan, rapporteure), $1^{\text {er }}$ février 2017, www.assembleenationale.fr/14/rap-info/i4428.asp
En matière de démantèlement nucléaire, la France se trouve actuellement dans une situation intermédiaire qui appelle à la plus grande vigilance compte tenu de l'ampleur des travaux qui restent à réaliser. Un démantèlement consiste en la déconstruction d'un réacteur nucléaire, la décontamination des bâtiments d'exploitation et l'assainissement des sols qui auraient pu être contaminés. En théorie et dans les cas les plus favorables, le démantèlement doit permettre la réutilisation sans contrainte des espaces libérés et entièrement décontaminés. On parle alors de " retour à l'herbe " ou de " champ vert ", l'image évoquant un retour à l'état de nature. Mais la réalité est plus complexe : la décontamination totale étant particulièrement onéreuse, l'Autorité de Sureté Nucléaire (ASN) peut accepter, dans certains cas et à la demande de l'exploitant que le démantèlement n'inclue pas cette contrainte. Aux États-Unis il est même admis que certains vestiges radioactifs puissent être laissés sur place recouverts d'un sarcophage; dans d'autres cas, les combustibles usagés peuvent être stockés sur des sites de réacteurs démantelés dans des silos étanches. Les Américains parlent alors d'une manière imagée de "champ marron ", adapté à une utilisation industrielle.

La France a opté pour le principe du démantèlement immédiat des installations après leur arrêt. Mais toutes les questions ne sont pas réglées concernant l'avancée des techniques de démantèlement et du retour d'expérience. À cet égard, il convient de faire observer deux points principaux : d'une part, les retours d'expérience sont inégaux selon les parcs ; d'autre part, la gestion des déchets issus des démantèlements pose encore question.

\section{Des retours d'expériences inégaux selon les parcs}

La spécificité du parc nucléaire français réside notamment dans l'existence d'un double parc : un premier parc ancien, à l'arrêt, constitué des réacteurs de première génération, dits « uranium naturel graphite gaz »(UNGG), et un second parc, plus récent, encore en activité, formé par les réacteurs à eau pressurisée (REP). EDF affirme connaitre des difficultés concernant le premier parc UNGG qui devait initialement être démantelé "sous eau ", et ces complications techniques ont amené EDF à reconsidérer sa stratégie. Alors que l'eau devait servir à limiter la libération de la radioactivité lors du retrait des couches de graphite, les principaux réacteurs voient leurs délais de démantèlement très largement repoussés. Ainsi, le réacteur de Bugey dont le démantèlement a débuté en 1994 devrait n'être démoli qu'en 2037, et celui de Chinon en 2041. L'ASN a cependant appris courant 2016 la décision d'EDF de procéder à un démantèlement sous air, sans que les justifications apportées par l'opérateur lui aient semblé satisfaisantes. En conséquence, EDF souhaite expérimenter sa nouvelle technique sur un réacteur test d'ici à 2060, et démanteler les réacteurs suivants d'ici à 2100 .

Concernant le parc REP, il apparait que la faisabilité technique est davantage assurée. Pour autant, dans les faits, aucun REP n'a été démantelé à ce jour dans le monde ; les surprises - mauvaises - en ce domaine étant jusque-là la règle, il convient d'être prudents. EDF compte 58 réacteurs à eau pressurisée actuellement en fonctionnement et neuf réacteurs à l'arrêt : Brennilis, réacteur à eau lourde, 


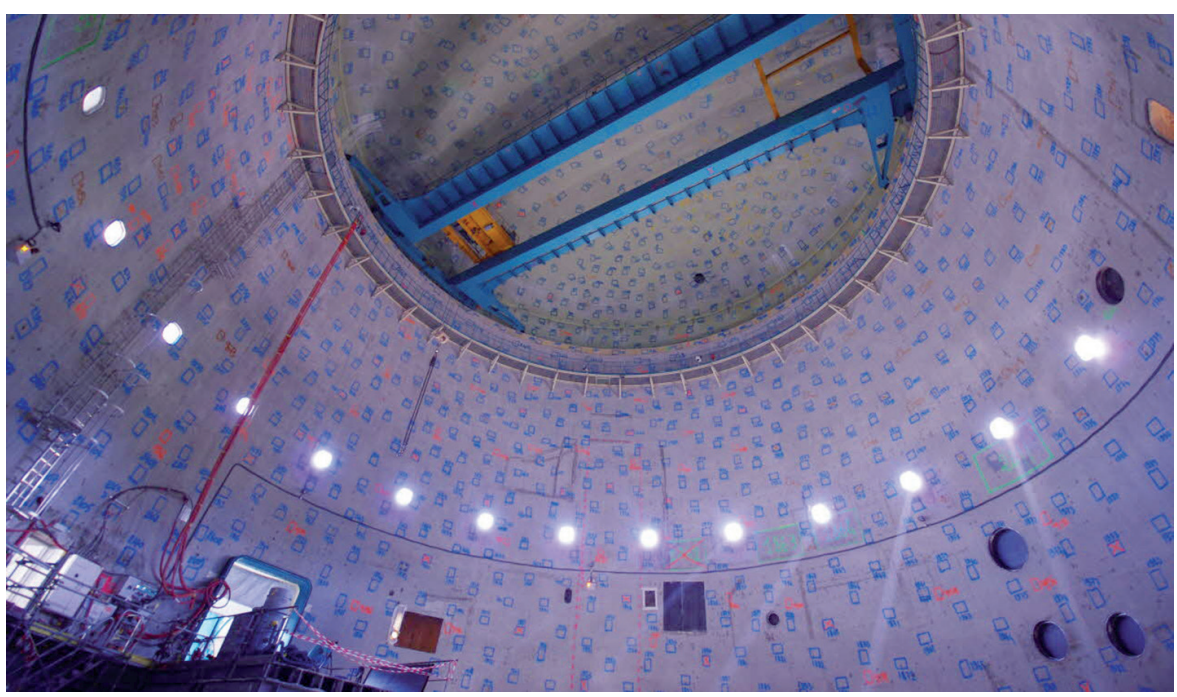

Superphénix fonctionnant au sodium, six réacteurs de première génération ayant fonctionné au graphite gaz, et le réacteur enterré de Chooz A, le plus ancien REP français. Sur ce parc, la difficulté tient davantage au projet d'EDF de reconstruire des réacteurs sur les sites actuellement utilisés. De ce fait, l'opérateur ne parle pas pour ces réacteurs de "démantèlement " mais de simple " déconstruction ". Autrement dit, EDF ne prévoit pas d'échéancier global et précis de leurs démantèlements. À cela s'ajoutent les difficultés ponctuelles de taille que constituent les réacteurs particuliers tels que Superphénix et Brennilis. Entré en service en 1985 et arrêté en 1996, Superphénix devrait être démantelé selon EDF à l'horizon 2028, soit plus de 30 ans après son arrêt définitif. En termes de mémoire de lieux, ce délai pose problème, de même qu'il ne respecte pas le principe du démantèlement immédiat. Brennilis, quant à lui, a été arrêté en 1985 et ne devrait pas être démantelé avant 2032 soit 47 ans après sa mise à l'arrêt - compte tenu des difficultés rencontrées par EDF. Or ces difficultés ont un réel impact financier : la Cour des comptes estime que les couts pourraient être multipliés par vingt, atteignant près de 482 milliards d'euros $^{(a)}$. La faisabilité technique du démantèlement est donc aussi une question financière.

On observe ainsi selon les types de réacteurs des disparités dans les savoirfaire qui ne permettent pas d'estimer acquise la faisabilité technique du démantèlement. Au-delà de la question des sites se pose celle de la gestion des combustibles usagés, condition essentielle pour le bon achèvement du démantèlement.

Réacteur expérimental Siloé (Grenoble), exploité de 1963 à 1997.

Les phases d'assainissement et de démantèlement ont duré de 1998 à 2011.

\section{La gestion des déchets pose encore question}

Là encore, la situation ne permet pas d'affirmer que les questions techniques sont en passe d'être réglées ${ }^{(b)}$. Si les déchets issus des démantèlements représentent à l'horizon 2030 près de $60 \%$ du volume des déchets à traiter, $40 \%$ seront issus de l'exploitation des réacteurs, dans un contexte de saturation de certains centres en fonction de la catégorie de déchets à traiter. Selon leur degré de radioactivité, tous les déchets ne subissent pas le même mode de retraitement et de stockage : $60 \%$ d'entre eux présentent une très faible activité (TFA), mais le centre de stockage de l'Agence nationale pour la gestion des déchets radioactifs (ANDRA) situé dans l'Aube arrivera à saturation en 2025.

La problématique du stockage pose, plus généralement, la question de la pertinence d'instaurer un seuil de libération pour les déchets issus du nucléaire. Actuellement, tout ce qui sort d'une centrale doit être stocké dans des centres spécialisés ; or certains déchets n'ont pas été contaminés et saturent donc les centres peut-être inutilement. L'ANDRA estime à cet égard que 30 à $50 \%$ des déchets ne présentent pas ou très peu de radioactivité. Il s'agirait donc d'une piste à étudier pour répondre au problème très prochain de la saturation de nos capacités de stockage.

L'autre motif d'inquiétude relative au stockage concerne ses modalités, et particulièrement le stockage en couches géologiques profondes. Le projet Cigéo situé à Bure dans la Meuse prévoit d'enfouir pour des centaines de milliers d'années les déchets les plus radioactifs de l'industrie nucléaire. Au vu de leurs conséquences à long terme et du caractère irréversible en pratique de ce choix ${ }^{(c)}$, le principe même de l'enfouissement est éminemment questionnable, alors même qu'il est possible de stocker ces déchets en subsurface et de poursuivre les recherches en parallèle pour espérer un jour pouvoir les recycler de manière satisfaisante. L'exemple d'une ancienne mine de sel en Basse-Saxe a montré les limites du stockage souterrain : les couloirs d'accès ne demeurent pas rectilignes à l'échelle d'une vie humaine ; comment espérer garantir la sécurité sur des milliers d'années? Toutes ces questions peuvent faire l'objet de choix stratégiques différents, mais encore faut-il ne pas prendre de décision irréversible, car le stockage des déchets, tout comme le démantèlement des réacteurs, constitue une étape décisive pour la réussite du démantèlement des infrastructures nucléaires. Force est de constater que là aussi, les perspectives actuelles sont insatisfaisantes.

a. Cour des comptes, Le cout de production de l'électricité nucléaire, actualisation mai 2014, www.ccomptes.fr.Voir aussi dans ce dossier l'article de A.-S. Dessillons (p. 29).

b. Sur cette question, voir plusieurs articles dans ce dossier, en particulier celui de J.-Y.Le Déaut (p.13), et l'entretien croisé avec C. Stéphan et P. Barbey (p. 19)

c. La loi n 2016-1015 du 25 juillet 2016 requiert en théorie une réversibilité, définie comme "la capacité, pour les générations successives, soit de poursuivre la construction puis l'exploitation des tranches successives d'un stockage, soit de réévaluer les choix définis antérieurement et de faire évoluer les solutions de gestion [...]. Elle inclut la possibilité de récupérer des colis de déchets déjà stockés. " [NdE]. 